\title{
Evaluación de la tinción de Hucker para la búsqueda rutinaria de Campylobacter sp en el estudio de un síndrome diarreico agudo*
}

\author{
LEONARDO CHANQUEO C., PATRICIA GARCÍA C., \\ EUGENIA LEÓN C. y ANTONIETA BLU F. ${ }^{3}$.
}

\section{Evaluation of Hucker stain as Campylobacter sp screening detection during an acute diarrhea disease*}

Campylobacter infection is one of the most frequent causes of gastroenteritis in the world and the third in Chile according to some studies. The routinary culture for Campylobacter in our country is not performed because of its high cost, and it is known, that the Hucker stain is a reasonable screening alternative. The objective of this study was to know the utility of the Hucker stain and estimate the frequency of Campylobacter in stool samples. A total of 5,750 stool samples received in the Catholic University Health Net Microbiology Laboratories, from March 2002 to May 2004, were studied with conventional stool culture and Hucker stain. In order to validate the Hucker stain with culture, during one month, all the stool samples were also studied with Campylobacter culture, with $35 \%$ sensitivity and $100 \%$ specificity. In $115 / 5.750$ samples (2\%), curved bacilli suggesting Campylobacter were observed by Hucker stain, and another 233 enteropathogens (4\%) which corresponded to: 151 Salmonella sp, 55 Shigella sp, 25 enterohemorrhagic E coli, and 2 Yersinia sp were isolated. When analyzing the patients in whom the Hucker stain was positive, $62.2 \%$ were younger than 5 years and of these, $63.8 \%$ were infants. We conclude that the Hucker stain is a simple and specific method, although not very sensitive, that allows us to increase the yield of diagnosing enteric pathogens in a 33\%. Campylobacter sp was in the second place after Salmonella sp in stool samples, and most frequent in young children. The active search for Campylobacter by means of culture is fundamental in the diagnosis of acute diarrhea.

Key words: Hucker stain, Campylobacter sp, Gastroenteritis.

Palabras claves: Tinción de Hucker, Campylobacter sp, Gastroenteritis.

\section{Introducción}

La infección por Campylobacter sp es una de las etiologías más comunes de gastroenteritis en el mundo, siendo en países desarrollados 2 a 7 veces más frecuente que infecciones por Salmonella sp, Shigella sp o Escherichia coli O157: H7 ${ }^{1}$. En E.U.A. se estiman alrededor de 2,4 millo- nes de infecciones por Campylobacter al año, asociadas con 13.174 hospitalizaciones y 124 muertes $^{2,3}$. En Latinoamérica la frecuencia relativa de aislamiento de Campylobacter sp en gastroenteritis aguda es de 5-20\% ${ }^{4}$ y en Chile, en estudio efectuados hace 20 años, ocupaba el tercer lugar entre las diarreas agudas de causa bacteriana en niños bajo 2 años de edad ${ }^{5,6}$.

Facultad de Medicina, Pontificia Universidad Católica de Chile:

Residente Infectología, Departamento de Medicina Interna. Programa de Enfermedades Infecciosas. (LCC)

Laboratorio de Microbiología. UDA Laboratorios Clínicos (PGC, ELC).

Estudiante de Medicina. Escuela de Medicina. (ABF)

* Artículo de ingreso a la Sociedad Chilena de Infectología (LCC)

Recibido: 5 enero 2005

Aceptado: 17 junio 2005 
El primer reconocimiento de la infección por Campylobacter fue reportado a principios del siglo XX, inicialmente conocido como Vibrio fetus (Campylobacter fetus), siendo agente causal de abortos sépticos en ovejas y vacuno. En 1947 V. fetus fue reportado como causa de aborto en mujeres y como patógeno oportunista en huéspedes inmunocomprometidos. En la década de los '70 se desarrollaron medios selectivos adecuados para su aislamiento, siendo reconocido desde los ' 80 como una causa importante de enfermedad gastrointestinal ${ }^{1}$.

El espectro clínico de la enteritis por Campylobacter va desde diarrea acuosa, sin sangre, a síndrome disentérico con dolor abdominal y fiebre, típicamente autolimitada e indistinguible de infecciones gastrointestinales causadas por otros enteropatógenos como Salmonella sp, Shigella sp y Yersinia sp. Se describen complicaciones locales que resultan de la propagación directa del tracto gastrointestinal (colecistitis, pancreatitis, peritonitis, etc.), extra intestinales (osteomielitis, endocarditis, etc.) y post infecciosas (síndrome de Guillain Barré, uveitis, anemia hemolítica, etc. $)^{1,7}$.

La infección por Campylobacter sp es usualmente esporádica, y se desarrolla por el consumo de alimentos mal cocidos, fundamentalmente de carne de pollo y otras especies ${ }^{8}$.

Campylobacter sp es un bacilo gramnegativo, curvo o en forma de $\mathrm{S}$ o gaviota de 0,2 - 0,9 $\mu \mathrm{m}$ de ancho y 0,5 - $5 \mu \mathrm{m}$ de largo, microaerófilo, no esporulado y móvil gracias a la presencia de un flagelo polar en uno o ambos extremos ${ }^{9}$. Se encuentra ampliamente distribuido en el ambiente, en una gran variedad de animales domésticos y silvestres, y en Chile se ha descrito hasta en $25,7 \%^{10}$ de las aves.

El género incluye varias especies, siendo $C$. jejuni subsp jejuni y $C$. coli los más frecuentemente aislados en infecciones humanas, aunque se reportan otras especies menos comunes como C. upsaliensis ${ }^{11}$, C. fetus y otros. Se ha descrito en Sudamérica y en Chile como patógeno emergente a $C$. jejuni subsp doylei, causante de diarrea secretora aguda ${ }^{12,13}$.

En nuestro país no se realiza el cultivo específico para Campylobacter en forma rutinaria dado el alto costo en el laboratorio; requiere de medios selectivos e incubación en microaerofilia a $42^{\circ} \mathrm{C}$. Se ha recomendado como alternativa de diagnóstico presuntivo rápido, de buen rendimiento y bajo costo, el uso de la tinción con cristal violeta de Hucker ${ }^{14}$, que de acuerdo a datos nacionales tiene una sensibilidad de $80 \%$ y valor predictor positivo de $56 \%{ }^{15}$.
El objetivo de este estudio fue evaluar la sensibilidad y especificidad de la tinción de Hucker en muestras de deposiciones y su utilidad como una estrategia de bajo costo en el diagnóstico de infección entérica por Campylobacter sp.

\section{Material y Métodos}

Se estudiaron en forma prospectiva todas las muestras de coprocultivos recibidas en el Laboratorio de Microbiología de la Red de Salud UC desde marzo del 2002 a mayo del 2004. En estas muestras, además del coprocultivo convencional se realizó la búsqueda de Campylobacter por tinción de Hucker, según lo descrito por Valenzuela y col ${ }^{15}$.

Las muestras se transportaron en medio Cary Blair (Eurotubo®, I.A.S.A, España) y fueron sembradas en agar Hektoen (Biomerieux ${ }^{\circledR}, \mathrm{Ha}$, MO), Mac Conkey (Biomerieux $\left.{ }^{\circledR}, \mathrm{Ha}, \mathrm{MO}\right) \mathrm{y}$ caldo selenito con resiembra en Hektoen después de incubar durante 16 horas a $35^{\circ} \mathrm{C}^{16}$. En muestras procedentes de pacientes bajo 10 años de edad se agregó siembra en agar Mac Conkey/ sorbitol (Laboratorios LINSAN®) .

Junto con la siembra en medios de cultivo, se realizó un frotis que fue teñido con partes iguales de cristal violeta de Hucker y bicarbonato de sodio al $1 \%$ durante uno a dos minutos. Las láminas fueron leídas con un aumento de 100x y se consideró positiva la presencia de formas espirilares semejantes a gaviotas. Si después de revisar 50 campos no se observaron estas formas espirilares, se consideró la lámina como negativa (Figura 1).

Por métodos convencionales ${ }^{17}$ se identificó Salmonella sp, Shigella sp y Yersinia sp. En muestras correspondientes a niños bajo 10 años de edad se agregó la búsqueda de E. coli entero-

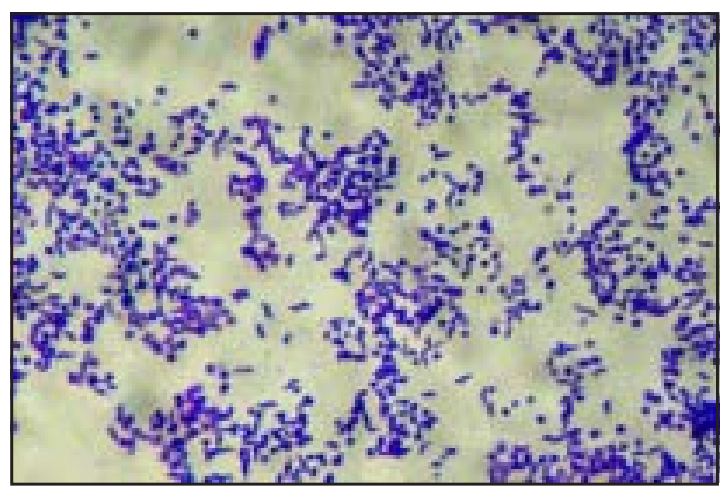

Figura 1. Bacilos curvos sugerentes de Campylobacter visualizados mediante tinción de Hucker. 
Tabla 1. Validación de la tinción de Hucker mediante cultivo de Campylobacter

\begin{tabular}{|c|c|c|c|c|}
\hline & & \multicolumn{3}{|c|}{ Cultivo de Campylobacter } \\
\hline & & Positivo & Negativo & Total \\
\hline Tinción & Positiva & 6 & 0 & 6 \\
\hline de & Negativa & 10 & 148 & 158 \\
\hline Hucker & Total & 16 & 148 & 164 \\
\hline
\end{tabular}

Sensibilidad: 6/16 = 37,5\%; Especificidad: 148/148 $=100,0 \%$

hemorrágica (ECEH) serotipos O26, O111, O157 y O55 utilizando antisueros PROBAC $®$ (Productos bacteriológicos LTDA, Brasil). Toda aglutinación positiva en lámina se confirmó en tubo, según las recomendaciones del Instituto de Salud Pública de Chile.

Dado el alto costo del cultivo de Campylobacter, fundamentalmente por el uso de agar selectivo y generador de microaerofilia $(\$ 3.000$ US 5.0) que se agregan al costo del coprocultivo convencional, no fue posible estudiar todas las muestras mediante cultivo. Por este motivo se diseñó una estrategia que permitiera validar la tinción de Hucker. Para esta validación se realizó en forma prospectiva y sólo durante un mes, cultivo de Campylobacter a todas las muestras de deposiciones recibidas en el laboratorio. Fueron sembradas en medio selectivo (agar Skirrow) y se incubaron a $42^{\circ}$ durante 48 horas en ambiente microaerófilo. La identificación de especie se realizó mediante tinción de Gram de la colonia, oxidasa e hidrólisis del hipurato.

Se revisó la edad de todos los pacientes cuyas muestras resultaron tener cultivos positivos.

\section{Resultados}

La validación de la tinción de Hucker con el cultivo de Campylobacter sp se obtuvo en 164 muestras de coprocultivos recibidas durante un mes en el Laboratorio de Microbiología. En 16 de estas muestras se cultivó Campylobacter, de los cuales sólo en 6 la tinción de Hucker fue positiva, mostrando una sensibilidad de $37,5 \%$ y especificidad de $100 \%$ (Tabla 1). Todos los aislamientos de Campylobacter correspondieron a la especie jejuni.

Del total de 5.750 muestras recibidas para coprocultivo, en 115 (2\%) se observaron bacilos curvos sugerentes de Campylobacter sp por tinción de Hucker; en ninguna de éstas hubo desarrollo de otros enteropatógenos. Además se recuperaron 233 enteropatógenos (4\%) que co-
Tabla 2. Enteropatógenos aislados en 5.750 muestras de deposiciones y rendimiento del coprocultivo

\begin{tabular}{lrl}
\hline Enteropatógenos & Total & $(\%)$ \\
\hline Salmonella sp & 151 & $(2,62 \%)$ \\
Enteritidis & 71 & \\
Montevideo & 23 & \\
Typhimurium & 30 & \\
Paratyphi B & 15 & \\
grupo B & 7 & \\
grupo D & 4 & \\
grupo E & 1 & \\
Shigella sp & 55 & $(0,95 \%)$ \\
S. sonnei & 44 & \\
S. flexneri & 11 & \\
E. coli enterohemorrágica & 25 & $(0,43 \%)$ \\
O26 & 15 & \\
O157 & 10 & \\
Yersinia enterocolitica & 2 & $(0,03 \%)$ \\
Total enteropatógenos & $233 \quad(4 \%)$ \\
Tinciones Hucker (+) & $115 \quad(2 \%)$ \\
Total & $348 \quad(6 \%)$ \\
\hline
\end{tabular}

rrespondieron a 151 Salmonella sp $(2,62 \%), 55$ Shigella sp $(0,95 \%), 25$ ECEH $(0,43 \%)$ y 2 Yersinia sp $(0,03 \%)$, cuyas especies se especifican en Tabla 2.

El promedio de edad de los pacientes con aislados de enteropatógenos en muestras de deposiciones fue de 22,5 años (rango 0 - 89 años) para Salmonella sp, 13,4 años (rango 0 - 78 años) para Shigella sp, 1,8 años (rango 0-7 años) para ECEH y de 1,5 (rango 0 a 3 años) para Yersinia sp. En los pacientes con tinción de Hucker positiva el promedio de edad fue 10,3 años (rango de 3 meses a 74 años), y 72 muestras $(62,6 \%)$ correspondieron a niños bajo 5 años de edad y de éstas $46(63,8 \%)$ a lactantes. 


\section{Discusión}

Dada la importancia clínica de la infección por Campylobacter sp en el hombre, es fundamental el aislamiento de este enteropatógeno en muestras de deposiciones; por esto en países con mayores recursos materiales como E.U.A. hasta 97\% de los laboratorios realizan en forma rutinaria búsqueda de Campylobacter sp en deposiciones ${ }^{18}$; en estos países se ha trabajado en las herramientas óptimas para la detección de Campylobacter sp, utilizando medios selectivos, medios selectivos con membranas de filtración, incubación en microaerofilia enriquecida con hidrógeno y técnicas de biología molecular con aplicación de RPC en deposiciones ${ }^{19-21}$. Los medios selectivos, con o sin membranas de filtración, serían los métodos óptimos para la detección de Campylobacter sp en muestras de deposiciones, aunque no permitirían el aislamiento de especies menos comunes como $C$. upsaliensis y C. hyointestinalis ${ }^{22}$. Las técnicas de RPC, que constituyen una metodología rápida y más sensible en la detección de especies menos comunes, son impracticables en países en desarrollo, por su alto costo y laboriosidad ${ }^{20}$.

En los laboratorios en que se realiza de rutina la detección de Campylobacter, el cultivo en medio selectivos en microaerofilia e identificación posterior por métodos fenotípicos es el método habitual. Debido al costo y demora del cultivo de Campylobacter, desde hace algunos años han surgido estrategias rápidas y de bajo costo para el diagnóstico precoz de éste patógeno, como la búsqueda directa con microscopia de contraste de fase y tinción de Gram, con sensibilidad de 43,5 a $65,5 \%$ y especificidad de 95 a $99,4 \%{ }^{23,24}$; un estudio más reciente arrojó una sensibilidad de $89 \%$, especificidad de $99,7 \%$ y valor predictor positivo de $97 \%{ }^{25}$. Otras tinciones desarrolladas, como la tinción con fucsina básica $1 \%{ }^{26}$ demuestra ser superior a la tinción de Gram con una sensibilidad de $94 \%$ y valor predictor positivo de $86 \%$, y la tinción con Cristal violeta de Hucker con una sensibilidad $80 \%{ }^{15}$.

La tinción de Hucker en nuestro estudio demostró una baja sensibilidad $(37,5 \%)$, con una especificidad de $100 \%$, siendo un método rápido, sencillo y de bajo costo. Sin embargo, es operador dependiente y no supera al cultivo para la búsqueda de este patógeno. Además es importante el aislamiento de Campylobacter sp en el estudio de un síndrome diarreico agudo, no sólo para conocer la especie involucrada, sino también para realizar los estudios de susceptibilidad, dada la resistencia creciente que ya se describe en algunos países frente a algunos antimicrobianos, como ciprofloxacina y eritromicina ${ }^{27-29}$.

La búsqueda activa de Campylobacter sp a través del cultivo es fundamental para el diagnóstico, permitiendo aumentar el aislamiento de patógenos entéricos. En nuestro estudio, a pesar de la baja sensibilidad de la tinción de Hucker, ésta permitió incrementar la detección de enteropatógenos en $33 \%$, aumentando el rendimiento global del método bacteriológico hasta en $6 \%$.

Este patógeno ocupa el segundo lugar después de Salmonella sp en las muestras de deposiciones recibidas para coprocultivo en nuestro laboratorio, concentrándose en edades tempranas de la vida, principalmente bajo los 5 años de edad. Si consideramos la baja sensibilidad de la tinción de Hucker, y la realización de rutina de coprocultivo con búsqueda de Campylobacter, este patógeno sería probablemente el más importante en el estudio de un síndrome diarreico agudo.

Nuestro trabajo demuestra, por tanto, que es necesario considerar la búsqueda rutinaria de Campylobacter sp en el estudio de un síndrome diarreico agudo, ya sea a través de la tinción de Hucker o, mejor aún, a través del cultivo. Este último, además de presentar una mejor sensibilidad que la tinción, permite determinar la especie y susceptibilidad de este enteropatógeno a los antimicrobianos.

Creemos que es importante conocer la real prevalencia de la infección por Campylobacter en nuestro medio en población adulta y pediátrica de forma prospectiva y con un adecuado grupo control.

\section{Resumen}

La infección por Campylobacter sp es una de las etiologías más comunes de gastroenteritis en el mundo, y en Chile ocuparía el tercer lugar entre las diarreas bacterianas en lactantes, según estudios previos. En nuestro país el cultivo no se realiza rutinariamente por su alto costo económico, siendo conocido que la tinción de Hucker puede ser una buena alternativa de pesquisa. El objetivo del estudio fue conocer la utilidad de la tinción de Hucker y estimar la frecuencia de Campylobacter sp en muestras de deposiciones. Se estudiaron 5.750 muestras de coprocultivos recibidas en el Laboratorio de Microbiología de la Red de Salud UC entre marzo del 2002 y mayo del 2004, en las cuales se realizó coprocultivo convencional y tinción de Hucker. Para la validación de la tinción de Hucker con el cultivo se sembró durante un mes todas las muestras para la búsqueda de Campylobacter sp, encontrando una sensibilidad de $37,5 \%$ y especificidad de 100\%. En 115/5.750 muestras (2\%) se observaron bacilos curvos sugerentes de Campylo- 
bacter sp por tinción de Hucker y se aislaron otros 233 entero patógenos $(4 \%)$ que correspondieron a: Salmonella sp (151), Shigella sp (55), Escherichia coli enterohemorrágica (25) y Yersinia sp (2). De las tinciones de Hucker positivas 62,6\% correspondieron a niños bajo 5 años y de éstas $63,8 \%$ a lactantes. Concluimos que la tinción de Hucker en un método sencillo y específico, aunque poco sensible, que permite aumentar el rendimiento de la detección microbiológica de patógenos entéricos en $33 \%$. En este estudio, Campylobacter sp ocupó el segundo lugar después de Salmonella sp en muestras de deposiciones enviadas para cultivo, concentrándose en edades tempranas de la vida. La búsqueda activa de Campylobacter sp por medio del cultivo específico es fundamental para el diagnóstico etiológico de un síndrome diarreico agudo.

\section{Referencias}

1.- Mishu B. Campylobacter jejuni Infections: Update on Emerging Issues and Trends. Clin Infect Dis 2001; 32: 1201-6.

2.- Mead P, Slutsker L, Dietz V, McCaig L, Bresee J, Shapiro C. Food-related illness and death in the United States. Emerg Infect Dis 1999; 5: 607-25.

3.- Samuel M, Vugia D, Shallow S, Marcus R, Segler S, McGivern $\mathrm{T}$ et al. Epidemiology of sporadic Campylobacter infection in the United States and declining trend in incidence, FoodNet 1996-1999. For Emerging Infections Program FoodNet Working Group. Clin Infect Dis 2004; 38: S165-74.

4.- Prado V, O’Ryan M. Acute gastroenteritis in Latin America. Infect Dis Clin North Am 1994; 8: 77-106.

5.- Prado V. Epidemiología de las enteritis por Campylobacter en niños. Rev Méd Chile 1984; 112: 1153.

6.- Prado V, Martínez J, Reyes L, Ducheylard M, Bercovich M, Millan V et al. Características de la infección intestinal por Campylobacter jejuni en lactantes chilenos. Rev Méd Chile 1985; 113: 521-5.

7.- Coker A, Isokpehi R, Thomas B, Amisu K, Obi L. Human Campylobacteriosis in developing countries. Emerg Infect Dis 2002; 8: 237-42.

8.- Friedman C, Hoekstra R, Samuel M, Marcus R, Bender J, Shiferaw B et al. Risk factors for sporadic Campylobacter infection in the United States: A casecontrol study in FoodNet Sites. Clin Infect Dis 2004; 38: S285-S296.

9.- Nachamkin I. Campylobacter and Arcobacter. Murray PR, Baron EJ, Jorgensen JH, Pfaller MA, Yolken RH, editors. Manual of Clinical Microbiology, $8^{\text {th }}$ ed. American Society of Microbiology. Washington DC; 2003, p. 902-914.

10.- Fernández H, Torres N. Campylobacter jejuni y Campylobacter coli en tres grupos de gallinas de diferente origen geográfico del sur de Chile. Arch Med Vet 2000; 32: 241-4.

11.- Labarca J, Sturgeon J, Borenstein L, Salem N, Harvey S, Lehnkering E et al. Campylobacter upsaliensis: Another pathogen for consideration in the United States. Clin Infect Dis 2002; 34: e59-e60.

12.- Fernández H, Fagundes Neto U, Ogatha S. Acute diarrhea associated with Campylobacter jejuni subsp doylei in Sao Paulo, Brazil. Pediatr Infect Dis J 1997;
16: 1098-9.

13.- Fernández H, Rodríguez R, Barudi C, Lobos M. A case of acute diarrhea due to the emerging pathogen Campylobacter jejuni subsp. doylei in Southern Chile. Braz J Microbiol 2003; 34: 52-4.

14.- Braun S, Camponovo R, Cona E, Fernández A, García P, González P, et al. Consenso. Síndrome diarreico agudo: Recomendaciones para el diagnóstico microbiológico. Rev Chil Infect 2002; 19: 101-13.

15.- Valenzuela M, D’Ottone K. Diagnóstico presuntivo rápido de enteritis asociada a Campylobacter. Rev Chil Infect 1984; 2: 132-4.

16.- Zimbro M J, Power D A. Difco \& BBL Manual. Manual of Microbiological Culture Media. BD; 2003, p. 508-9.

17.- Farmer J J III. Enterobacteriaceae: Introduction and Identification. Murray PR, Baron EJ, Jorgensen JH, Pfaller MA, Yolken RH, editors. Manual of Clinical Microbiology, $8^{\text {th }}$ ed. American Society of Microbiology. Washington DC; 2003, p. 636-53.

18.- Voetsch A, Angulo F, Rabatsky-Ehr T, Shallow S, Cassidy M, Thomas S et al. Laboratory practices for stool-specimen Culture for bacterial pathogens, including Escherichia coli O157:H7, in the FoodNet Sites, 1995-2000. Clin Infect Dis 2004; 38: S190-7.

19.- Lastovica AJ, Le Roux E. Efficient isolation of Campylobacteria from stools. J Clin Microbiol 2000; 38: 2798-9.

20.- Lastovica A, Le Roux E. Optimal detection of Campylobacter spp in stools. J Clin Pathol 2003; 56: 480.

21.- Maher M, Finnegan C, Collins E, Ward B, Carroll C, Cormican M. Evaluation of culture methods and a DNA probe-based PCR assay for detection of Campylobacter species in clinical specimens of feces. J Clin Microbiol 2003; 41: 2980-6.

22.- Kulkarni S, Lever S, Logan J, Lawson A, Stanley J, Shafi M. Detection of Campylobacter species: a comparison of culture and polymerase chain reaction based methods. J Clin Pathol 2002; 55: 749-53.

23.- Ho D, Ault M, Ault M, Murata G. Campylobacter enteritis. Early diagnosis with Gram's stain. Arch Intern Med 1982; 142: 1858-60.

24.- Sazie E, Titus A. Rapid diagnosis of Campylobacter enteritis. Ann Intern Med 1982; 96: 62-3.

25.- Wang H, Murdoch D. Detection of Campylobacter species in faecal samples by direct Gram stain microscopy. Pathology 2004; 36: 343-4.

26.- Park C, Hixon D, Polhemus A, Ferguson C, Hall S, Risheim $\mathrm{C}$ et al. A rapid diagnosis of Campylobacter enteritis by direct smear examination. Am J Clin Pathol 1983; 80:388-90.

27.- Engberg J, Neimann J, Moller E Nielsen, Moller F, Fussing V. Quinolone-resistant Campylobacter infections: Risk factors and clinical consequences. Emerg Infect Dis 2004; 10: 1056-63.

28.- Gupta A, Nelson J, Barrett T, Tauxe R, Rossiter S, Friedman $\mathrm{C}$ et al. Antimicrobial resistance among Campylobacter strains, United States, 1997-2001. Emerg Infect Dis 2004; 10: 1102-9.

29.- Guerrant R, Van Gilder T, Steiner T, Thielman N, Slutsker L, Tauxe R et al. Practice guidelines for the management of infectious diarrhea. Clin Infect Dis 2001; 32: 331-51.

Correspondencia a:

Patricia García C.

pgarcia@med.puc.cl 